\title{
PRESENCIA DE PROTEROTHERIIDAE (MAMMALIA, LITOPTERNA) EN EL PLEISTOCENO DE LAS PAMPAS BONAERENSES
}

\author{
FEDERICO L. AGNOLIN (1) \\ Laboratorio de Anatomía Comparada y Evolución de los Vertebrados, Museo Argentino de Ciencias Naturales "Bernardino \\ Rivadavia", Av. Ángel Gallardo 470, C1405DJR; Fundación de Historia Natural "Félix de Azara", Departamento de \\ Ciencias Naturales y Antropología, Universidad Maimónides, Hidalgo 775, C1405BDB, Buenos Aires, Argentina. \\ fedeagnolin@yahoo.com.ar \\ NICOLÁS R. CHIMENTO (1) \\ Laboratorio de Anatomía Comparada y Evolución de los Vertebrados, Museo Argentino de Ciencias Naturales "Bernardino \\ Rivadavia", Av. Ángel Gallardo 470, C1405DJR, Buenos Aires, Argentina. \\ nicochimento@hotmail.com \\ MARCELO R. BRUYERE (D) \\ Lautaro 277, C1406, Buenos Aires, Argentina. \\ marcelo.bruyere@gmail.com
}

\begin{abstract}
Presence of Proterotheriidae (Mammalia, Litopterna) in the Pleistocene of the Bonaerian Pampas. The fossil record of Pleistocene Proterotheriidae is scarce. It is restricted to the species Neolicaphrium recens known from Brazil, Uruguay, and Argentina, and to Uruguayodon alius, from Uruguay. Up to the date, proterotheriids are absent from the Pleistocene record of the Pampean Region. The aim of the present contribution is to report isolated remains of proterotheriids coming from Punta Indio and Punta Médanos localities, at the coast of Buenos Aires Province, Argentina. The specimens were found in the coastline and lack precise stratigraphical position. These findings indicate that Pleistocene proterotheriids were probably more diversified and geographically widespread than previously thought.
\end{abstract}

Keywords: Punta Piedras, Punta Medanos, Pampean Region, Buenos Aires Province, Neolicaphrium.

RESUMO - O registro fóssil pleistocênico de Proterotheriidae é escasso. Se restringe às espécies Neolicaphrium recens, conhecida para o Brasil, Uruguai e Argentina, e Uruguayodon alius do Uruguai. Até o presente momento, os proteroterídeos estão ausentes no Pleistoceno da Região Pampeana da Argentina. A presente contribuição tem por objetivo reportar restos isolados de proteroterídeos provenientes das localidades de Punta Indio e Punta Médanos, região costeira da Província de Buenos Aires, Argentina. Os espécimes foram encontrados na linha de costa e carecem de contexto estratigráfico preciso. Estes achados sugerem que os proteroterídeos pleistocênicos eram possivelmente mais diversificados e mais amplamente distribuídos geograficamente do que se supunha anteriormente.

Palavras-chave: Punta Piedras, Punta Medanos, Región Pampeana, Província de Buenos Aires, Neolicaphrium.

\section{INTRODUCCIÓN}

Proterotheriidae (Litopterna) es una de las familias de ungulados endémicos de América del Sur con mayor cantidad de especies reconocidas (Bond et al., 1995; Croft et al., 2020). Los proterotéridos fueron herbívoros de tamaño pequeño a mediano, con tendencia a la monodactilia (reducción de dedos II y IV, pérdida de los dedos I y V, y desarrollo del dedo III), adquirida de manera convergente a los caballos actuales (Soria, 2001).

Aunque han sido citados para el Paleoceno, registros seguros de la familia se remontan al Oligoceno (Soria, 2001). Desde su reconocimiento por Ameghino (1887), los Proterotheriidae se consideraron extintos en el Plioceno.
Frenguelli (1921) describió en gran detalle una mandíbula procedente del Pleistoceno de la Provincia de Córdoba. Esta mandíbula constituyó el holotipo (y único material conocido) de la especie Neolicaphrium recens Frenguelli, 1921, por aquel entonces considerado el único Proterotheriidae registrado en tiempos post-pliocenos. Sin embargo, Kraglievich (1934) consideró que los restos de Neolicaphrium eran el resultado de una mezcla de fósiles, y en consecuencia provendría de sedimentos pliocénicos, un criterio seguido por la gran mayoría de los autores posteriores. Nuevos hallazgos en Uruguay (Perea et al., 1995) sugirieron que Neolicaphrium efectivamente procedía de sedimentos pleistocénicos, lo que fue corroborado por Bond et al. (2001) quienes registraron diversos restos de esta especie procedentes del centro y 
litoral argentinos, así como de Uruguay. Pronto, la especie fue ampliamente reportada para el norte de Uruguay, sur de Brasil y centro y este de Argentina, siempre fuera de la Región Pampeana (Tauber, 2000; Bond et al., 2001; Scherer et al., 2009; Vezzosi et al., 2009; Ubilla et al., 2011; Luna et al., 2015; Schmidt et al., 2016; Vezzosi, 2016; Gaudioso et al., 2017; Corona et al., 2019a, b). De hecho, en Argentina se la reportó para las provincias de Córdoba (Frenguelli, 1921; Tauber, 2000; Schmidt et al., 2016), Santiago del Estero (Gaudioso et al., 2017), Corrientes (Álvarez, 1974; Bond et al., 2001) y Santa Fe (Vezzosi et al., 2009; Ubilla et al., 2011). A pesar del notable incremento de registros ocurrido a lo largo de la última década, los fósiles atribuidos a Neolicaphrium recens son escasos y la mayor parte de ellos corresponden a restos mandibulares o dientes aislados (pero véase Ubilla et al., 2011; Schmidt et al., 2016; Corona et al., 2019b; Ghizzoni et al., 2020). Más recientemente, Corona et al. (2019a) describieron para el Pleistoceno Medio de Uruguay el taxón Uruguayodon alius Corona, Perea \& Ubilla, 2019, basados en restos mandibulares y postcraneanos. Este hallazgo constituye el segundo Proterotheriidae conocido para el Pleistoceno. La finalidad de la presente contribución es la de dar a conocer la presencia de Proterotheriidae en dos localidades del Pleistoceno de la región pampeana y explorar las implicancias paleobiogeográficas de dichos hallazgos.

\section{POSICIÓN GEOGRÁFICA Y ESTRATIGRÁFICA}

Los ejemplares MLP 17-II-18-23 y MLP 17-II-18-25 han sido colectados en la costa del Río de La Plata, en la localidad de Punta Indio, Partido de Punta Indio, Provincia de Buenos Aires, Argentina (Figura 1A). Los ejemplares fueron hallados rodados en la línea de costa, en conjunto con megafauna como Mesotherium cristatum (Serres, 1867), Ctenomys latidens (Gervais \& Ameghino, 1880), Conepatus primaevus Burmeister, 1864 y Glyptodon munizi Ameghino, 1881. Estos taxones son considerados como fósiles típicos del piso Ensenadense (Pleistoceno Inferior-Medio; Cione \& Tonni, 2005; Soibelzon et al., 2008). Pomi (2008) relevó

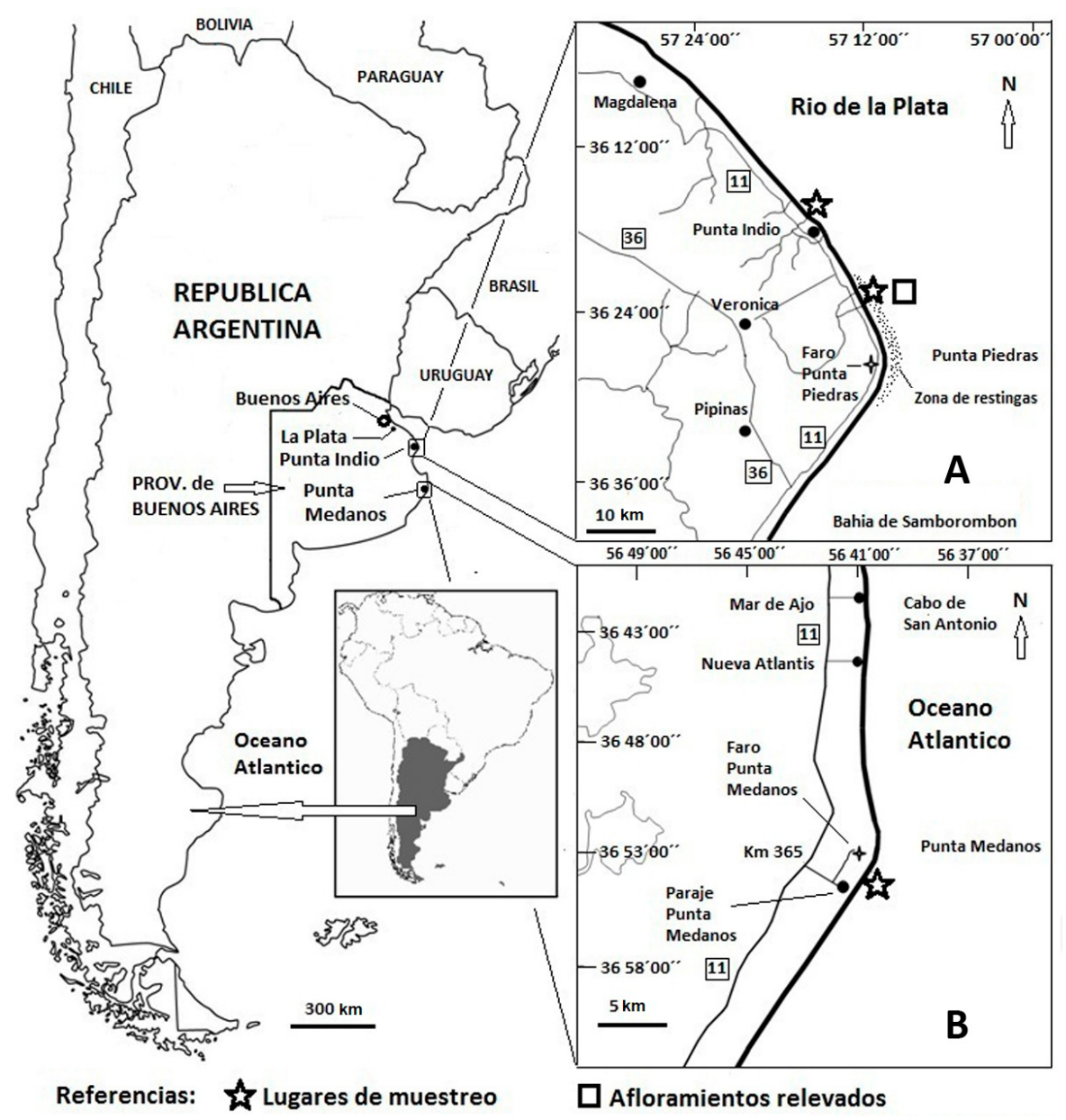

Figura 1. Ubicación de las localidades mencionadas en el texto. A, detalle de la costa noreste de la Provincia de Buenos Aires, donde se encuentra el partido de Punta Indio. B, detalle de la costa sudeste de la Provincia de Buenos Aires, con la ubicación de la localidad de Punta Médanos. Las estrellas indican la posición de los materiales colectados, el cuadrado indica la posición de los estratos ilustrados en la Figura 2.

Figure 1. Locations mentioned in the text. A, detail of the northeast coast of the Province of Buenos Aires, where the Punta Indio district is located. B, detail of the southeast coast of the Province of Buenos Aires, with the location of the town of Punta Médanos. The stars indicate the position of the collected materials, the square indicates the position of the layers illustrated in Figure 2. 
los restos mastofaunísticos procedentes del lecho y costa del Río de La Plata en las inmediaciones de Punta Indio. Este autor indicó que los sedimentos fosilíferos en esta localidad forman una gran restinga de varios kilómetros de extensión (expuesta sólo en condiciones de bajas mareas) que consiste en limos arcillosos pardo-rojizos y limos arenosos, con abundantes concreciones laminares de carbonato de calcio (ver Figura 2). Sobre la base de su contenido fosilífero, Pomi (2008) asignó dichos estratos al Ensenadense (Pleistoceno Inferior-Medio).

Vale la pena remarcar que la costa del Río de La Plata desde Punta Indio a Punta Piedras no parece tener rastros de otros sedimentos del Pleistoceno y Holoceno Inferior que no sean aquellos pertenecientes a la Formación Ensenada. En este sentido, la observación en Punta Indio y Punta Piedras de amplios afloramientos durante bajantes excepcionales del río (con el agua a $1 \mathrm{~m}$ por debajo de los valores astronómicos de tablas de marea; Figura 2) muestran que los estratos de la Formación Ensenada subyacen directamente a sedimentos del Holoceno Tardío. Ambas unidades están separadas por una discontinuidad neta y erosiva, no existiendo rastros de otras unidades estratigráficas.

En consecuencia, debido a que sedimentos pertenecientes a la Formación Ensenada están presentes y son de amplia extensión en el área aquí tratada, los ejemplares MLP 17-II18-23 y MLP 17-II-18-25 son tentativamente asignados a una edad Ensenadense.

El ejemplar MMA 0050 procede de la costa marina de la localidad de Punta Médanos, en el Partido de La Costa, Provincia de Buenos Aires, Argentina (Figura 1B). El ejemplar fue hallado rodado suelto en la línea de costa, en conjunto con taxones típicos de la megafauna pampeana, incluyendo Glyptodon sp., Scelidotherium sp., Lama sp. y Hemiauchenia sp.

Restos fósiles rodados y ex situ de diversos megamamíferos han sido recolectados de manera frecuente a lo largo de la planicie costera del Partido de La Costa (Tonni \& Cione, 1999; Cione et al., 2005). Tonni \& Cione (1999) consideraron en detalle la posible procedencia estratigráfica de los ejemplares hallados en la costa, y si bien la procedencia estratigráfica

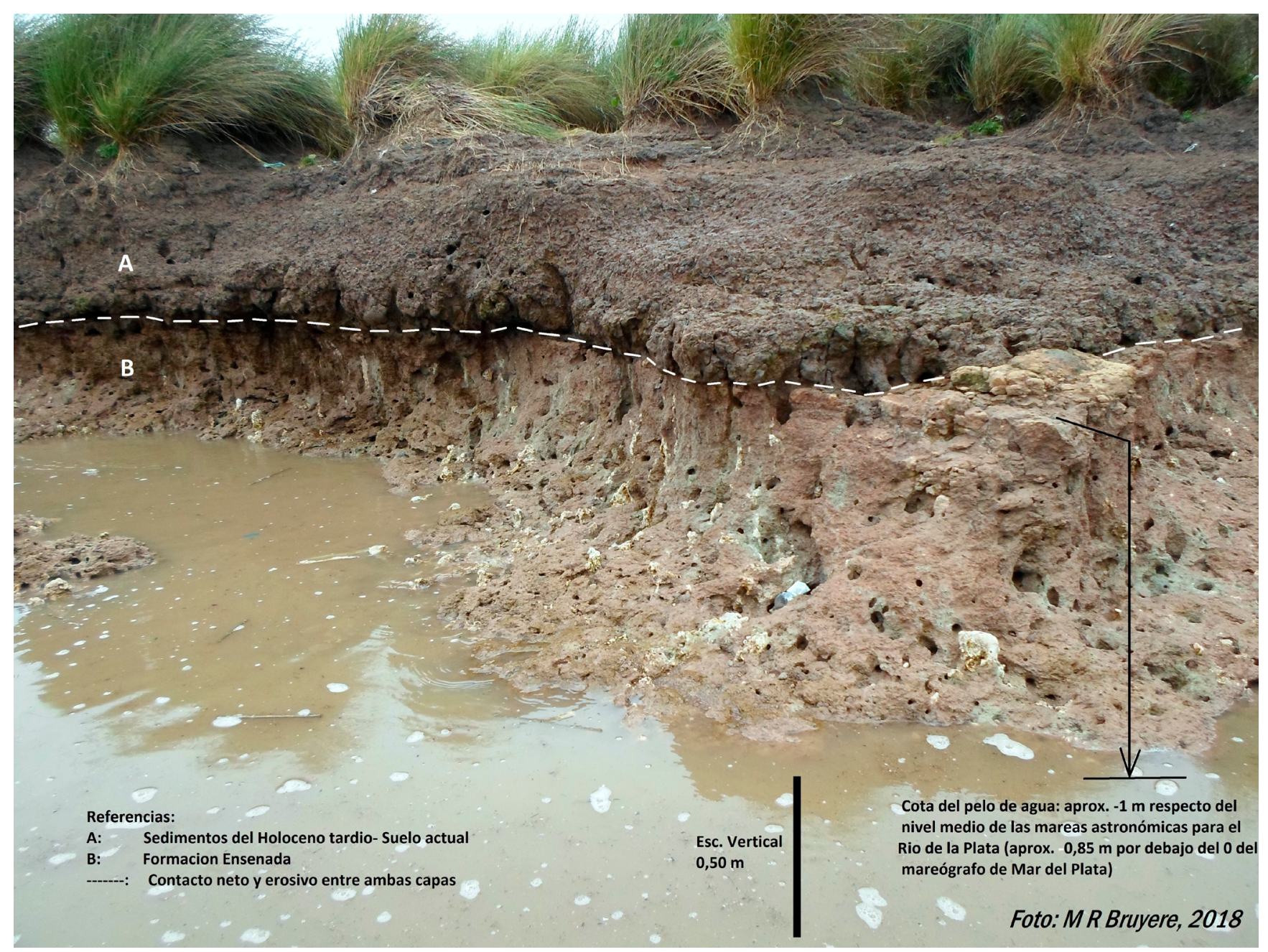

Figura 2. Fotografía de la región costera del Río de La Plata en la localidad de Punta Piedras en una bajante excepcional del nivel del río. Se observan los afloramientos de la Formación Ensenada (por debajo) en neta discordancia erosiva con las capas suprayacentes pertenecientes al Holoceno Tardío.

Figure 2. Photograph of the coastal region of the Río de La Plata in the locality of Punta Piedras in an exceptional downspout of the river level. The outcrops of the Ensenada Formation (below) are observed in clear erosive discordance with the overlying layers belonging to the Late Holocene. 
exacta no es clara, estos autores señalan con alta probabilidad que provendrían de la Formación Pozo 10, cuya antigüedad ronda los 120.000 años antes del presente (Violante \& Parker, 2004). En consecuencia, asignamos tentativamente MMA 0050 a dicha unidad estratigráfica.

\section{MATERIAL Y MÉTODOS}

Los materiales fueron descritos siguiendo la terminología de Soria (2001), Schmidt (2015) y Carrillo et al. (2018). Se sigue la sistemática de Bond et al. (2001) y Soria (2001).

Abreviaturas institucionales: CFA MA, Colección de Mastozoología, Fundación de Historia Natural "Félix de Azara", Buenos Aires, Argentina; MACN A-, Colección Nacional Ameghino, Museo Argentino de Ciencias Naturales "Bernardino Rivadavia", Buenos Aires, Argentina; MACNMa, Colección Mastozoología, Museo Argentino de Ciencias Naturales "Bernardino Rivadavia", Buenos Aires, Argentina; MACN Pv-, Colección Paleontología de Vertebrados, Museo Argentino de Ciencias Naturales "Bernardino Rivadavia", Buenos Aires, Argentina; MLP, Colección Paleontología de Vertebrados, Museo de La Plata, La Plata, Provincia de Buenos Aires, Argentina; MMA, Museo Municipal de Mar de Ajó, Mar de Ajó, Provincia de Buenos Aires, Argentina.

\section{PALEONTOLOGÍA SISTEMÁTICA}

\author{
MAMMALIA Linnaeus, 1758 \\ LITOPTERNA Ameghino, 1889 \\ LOPHOLIPTERNA Cifelli, 1983 \\ PROTEROTHERIIDAE Ameghino, 1887
}

Gen. et sp. indet.

Material referido. MLP 17-II-18-23, posible $\mathrm{m} 1$ derecho con el lóbulo mesial incompleto (Figura 3); MMA 0050, astrágalo izquierdo con las caras medial y lateral erodadas y la faceta sustentacular incompleta (Figuras 4A-F); MLP 17-II-18-25, astrágalo izquierdo (Figuras 4G-L).

Descripción. Los ejemplares aquí descriptos son coincidentes en pertenecer a una especie de Proterotheriidae, algo mayor que un ciervo de las pampas (Ozotoceros bezoarticus Linnaeus, 1758). El $\mathrm{m} 1$ es de corona relativamente baja y presenta la superficie oclusal muy desgastada que ha obliterado la mayor parte de sus rasgos anatómicos. Las raíces no se han preservado. En vista oclusal es de contorno subrectangular y exhibe restos de cemento. Ectofléxido profundo y estrecho, de forma subtriangular, penetrando aproximadamente hasta la mitad del ancho transverso del molar, y perpendicular al eje mesiodistal del diente. Hipoconúlido e hipolofúlido bien desarrollados. El hipocónido es de contorno redondeado, más ancho mesiodistalmente que el protocónido. No hay evidencias de entofléxido o metafléxido ni cíngulo lingual. El borde lingual es más alto que el resto de la corona. Las capas de esmalte mesial y distal se encuentran adelgazadas, debido a la presión que ejercen las piezas adyacentes, resultando en una condición dentaria imbricada (Ghizzoni et al., 2020). Ambos astrágalos (MLP 17-II-18-25; MMA 0050) son indiferenciables en sus rasgos anatómicos principales. Comparten la presencia de un cuerpo alargado y estrecho, con la tróclea en forma de polea, el surco medio profundo, flanqueado por fuertes crestas verticales subparalelas al eje mayor del hueso; la cresta lateral se encuentra más desarrollada que la medial, el cuello astragalar alargado y estrecho, con una amplia y profunda fosa en la base, cabeza astragalar conformada casi exclusiva por una faceta navicular, la cual se proyecta dorsalmente, cara ventral con la faceta sustentacular amplia y convexa, continua con la superficie navicular, faceta ectal de contorno subtriangular en vista plantar, profundamente excavada (implicando un amplio contacto con el calcáneo), faceta fibular amplia y plana, y la faceta maleolar fuertemente cóncava. El astrágalo MMA 0050 presenta ciertos rasgos que lo distinguen de MLP 17-II18-25. La cabeza astragalar es suavemente convexa en MLP 17-II-18-25, y plana distalmente en MMA 0050. El margen lateral es recto próximodistalmente en MMA 0050, mientras que en MLP 17-II-18-25 el cuello se proyecta lateralmente,

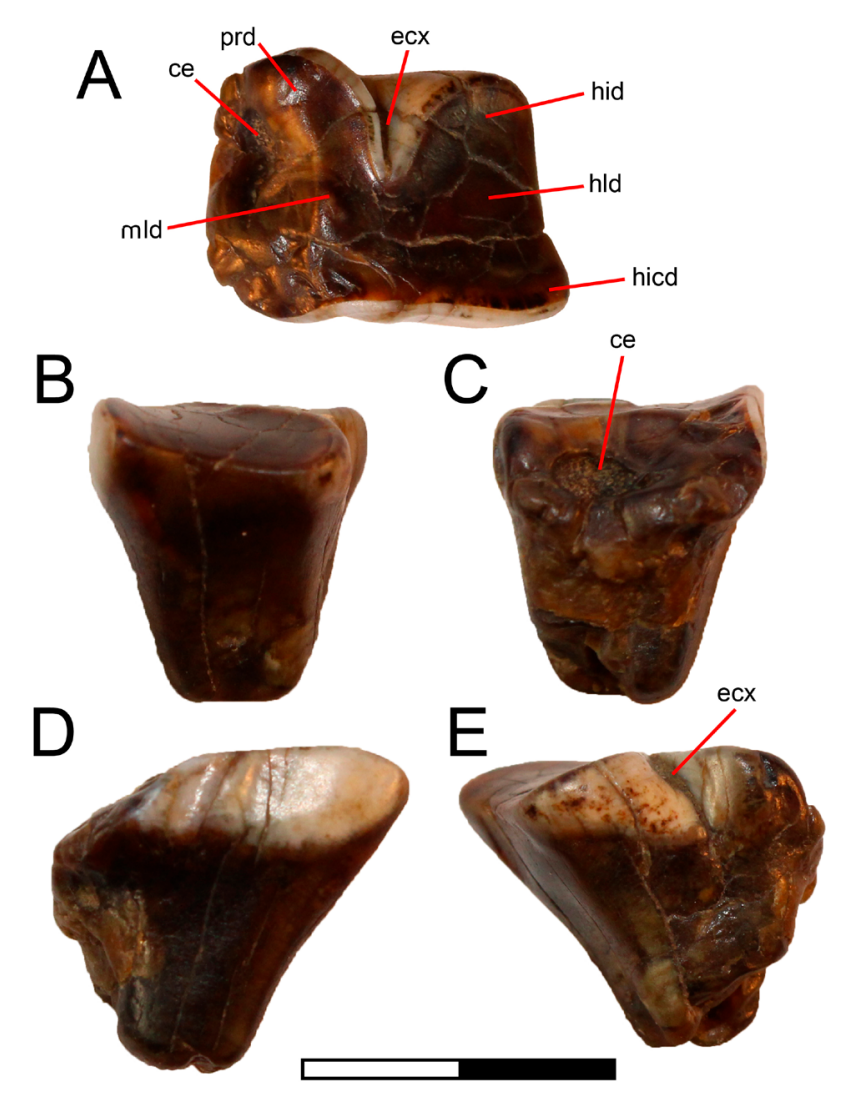

Figura 3. MLP 17-II-18-23, posible $m 1$ derecho en vista oclusal (A), distal (B), mesial (C), lingual (D) y labial (E). Abreviaturas: ce, cemento; ecx, ectofléxido; hicd, hipoconúlido; hid, hipocónido; hld, hipolofúlido; mld, metalófido; prd, protocónido. Escala $=10 \mathrm{~mm}$.

Figure 3. MLP 17-II-18-23, possible right $\mathrm{m} 1$ in occlusal (A), distal (B), mesial (C), lingual (D) and labial (E) views. Abbreviations: ce, cement; ecx, ectoflexid; hicd, hypoconulid; hid, hypoconid; hld, hypolophulid; mld, metalophid; prd, protoconid. Scale bar $=10 \mathrm{~mm}$. 

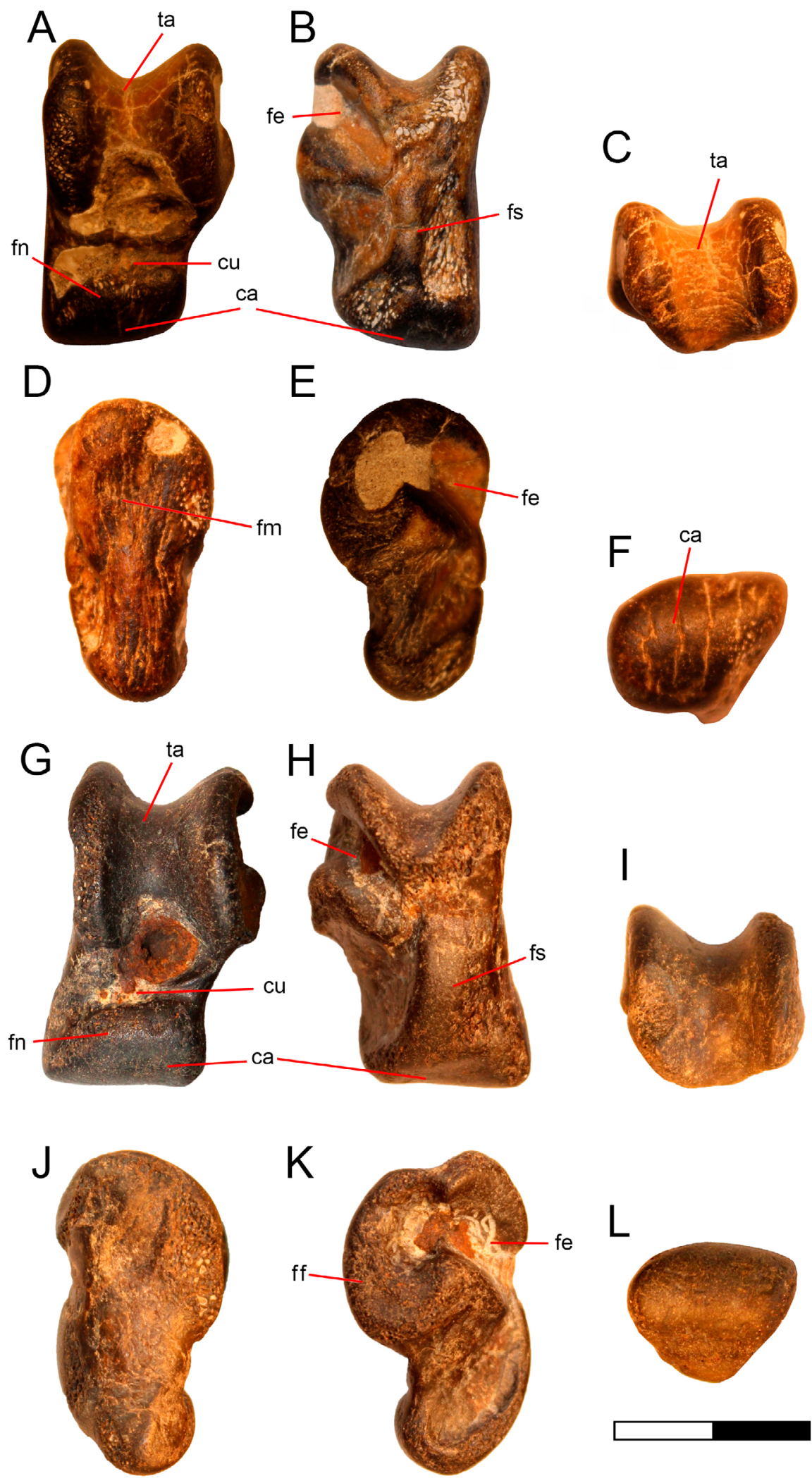
resultando en un margen lateral sigmoideo. MMA 0050 presenta signos de mayor transporte, como ser los bordes más redondeados e incrustaciones de tosca, por lo cual es posible que las diferencias notadas entre ambos astrágalos se deban a diferentes procesos tafonómicos.

\section{DISCUSIÓN}

\section{Ubicación taxonómica de los ejemplares descriptos}

La siguiente combinación de rasgos permite referir los ejemplares aquí descriptos a Proterotheriidae: $\mathrm{m} 1$ bicrescéntico (es decir compuesto por dos lóbulos en forma de media-luna) y lofoselenodonte, fuertemente imbricado a otros molariformes (como lo evidencia el desarrollo del hipoconúlido e hipolófido), astrágalo transversalmente estrecho y columnar, con cuello alargado y comprimido, tróclea distal en forma de polea con crestas muy profundas y bien desarrolladas y cabeza astragalar subcilíndrica con faceta navicular extendida dorsal y ventralmente (Scott, 1910; Cifelli, 1983; Bond et al., 2001; Soria, 2001; Figuras 3, 5).

Los molares desgastados de MLP 17-II-18-23, Neolicaphrium y Uruguayodon (Bond et al., 2001; Corona et al., 2019b), comparten la presencia de cemento en la corona, la forma subcuadrangular y la delgadez del esmalte en los bordes mesial y distal del $\mathrm{m} 1$. La ausencia de entofléxido en el borde lingual del $\mathrm{m} 1$ asemeja el material descrito a algunos proterotéridos de edades Colhuehuapense y Santacrucence, como Lambdaconus, Prolicaphrium, Tetramerorhinus, Anisolophus, entre otros (ver Soria, 2001). Sin embargo, este rasgo es variable a lo largo del desgaste de los molares (Soria, 2001). Por otro lado, en MLP 17-II-18-23 el ectofléxido es muy profundo, y separa profundamente ambos lóbulos de la corona, a diferencia de los géneros antes mencionados donde apenas penetra el diente, en el caso de los molares bien desgastados. El m1, cuando es comparado con molares igualmente desgastados de Neolicaphrium, se distingue por carecer de entofléxido (Bond et al., 2001; Gaudioso et al., 2009). La ausencia de todo vestigio de cíngulo entofléxido o metafléxido y el ectofléxido profundo y estrecho en MLP 17-II-18-23, lo diferencian de Uruguayodon (Corona et al., 2019a). Debido a su combinación de rasgos este ejemplar no puede ser asignado a los géneros de Proterotheriidae pleistocénicos conocidos. En consecuencia, es determinado aquí como Proterotheriidae indet.

Dentro de Proterotheriidae, la conformación del astrágalo es muy homogénea entre los diferentes géneros, y exhibe una variación intraespecífica pronunciada en la mayor parte de los caracteres, por lo que no resulta un elemento de gran utilidad taxonómica (Soria, 2001). Sin embargo, los astrágalos aquí descriptos difieren de Proterotheriidae terciarios (e.g. Tetramerorhinus, Diadiaphorus, Epecuenia) en el cuello transversalmente estrecho, ausencia de inclinación en las crestas de la tróclea y en la cabeza levemente orientada en sentido medial (Soria, 2001; Scherer et al., 2009). En todos estos rasgos los especímenes son congruentes con un ejemplar determinado como cf. Neolicaphrium recens del Pleistoceno de Brasil por Scherer et al. (2009).
Scherer et al. (2009) asignaron restos postcraneanos hallados rodados en la costa marina de Brasil, a Neolicaphrium, basados en el tamaño relativamente pequeño. Sin embargo, dado que los materiales no fueron encontrados en asociación a restos craneanos con dientes, esta asignación es dudosa. En consecuencia, los materiales descriptos por Scherer et al. (2009) son aquí considerados como Proterotheriidae indeterminados. Del mismo modo, los astrágalos aquí reportados no son determinados más allá del nivel familiar.

\section{Diversidad y distribución de Proterotheriidae en el Pleistoceno sudamericano}

Los Proterotheriidae constituyeron un clado relativamente exitoso y diversificado a lo largo de gran parte del Cenozoico, desde el Oligoceno (Soria, 2001) hasta el Mioceno tardío, con algunos registros más escasos Plio-Pleistocénicos (Villafañe et al., 2006). Para el Chapadmalense (Plioceno temprano) se encuentran representados por la especie Neolicaphrium major Soria, 2001, mientras que para el Pleistoceno, se contaba hasta la década de 2000 con la especie Neolicaphrium recens, del Ensenadense y Lujanense de las provincias de Córdoba, Entre Ríos, Corrientes, Santa Fe, Santiago del Estero y los países limítrofes de Uruguay y Brasil (Tauber, 2000; Bond et al., 2001; Scherer et al., 2009; Vezzosi et al., 2009; Ubilla et al., 2011; Gaudioso et al., 2017; Corona et al., 2019a). Debido a que solo se reconocía una especie para el Pleistoceno y a que sus restos eran relativamente escasos, Bond et al. (2001) lo consideraron un taxón relictual, un criterio compartido por otros autores (Villafañe et al., 2006; Scherer et al., 2009). Más aún, debido a que sus registros se ubicaban en bajas latitudes y rodeaban la región pampeana, se propuso que los Proterotheriidae del Pleistoceno serían formas adaptadas a climas relativamente cálidos y ambientes con vegetación arbustiva o arbolada esparcida, posiblemente tipo sabana, diferente de la imperante en la región pampeana (Bond et al., 2001; Ubilla et al., 2011; Corona et al., 2019a). Más aún, algunos autores han inferido hábitos ramoneadores para $N$. recens (Bond et al., 2001; Corona et al., 2019b). Por otro lado, Ghizzoni et al. (2020) sostienen que Neolicaphrium y Uruguayodon podrían haber sido de hábitos pastadores.

El registro de al menos tres taxones diferentes (Neolicaphrium, Uruguayodon, y MLP 17-II-18-23), indica que durante el Pleistoceno los Proterotheriidae posiblemente se encontraban más diversificados que lo supuesto con anterioridad. De esta manera, su condición relictual es dudosa, y es posible que mayores exploraciones y la revisión exhaustiva de colecciones puedan arrojar materiales adicionales que sustenten la coexistencia de varios Proterotheriidae durante el Pleistoceno del Cono Sur.

El hallazgo de diversos materiales de Proterotheriidae en la región pampeana también cuestiona seriamente la hipótesis que sostenía que los Proterotheriidae del Pleistoceno se habrían encontrado ausentes en la región pampeana debido a requerimientos ecológicos específicos, como ser afinidades a ambientes con mayores temperaturas y humedad, y a fisonomías de bosques y/o arboledas (Bond et al., 2001; Villafañe et al., 2006; Scherer et al., 2009; Ubilla et al., 2011; 

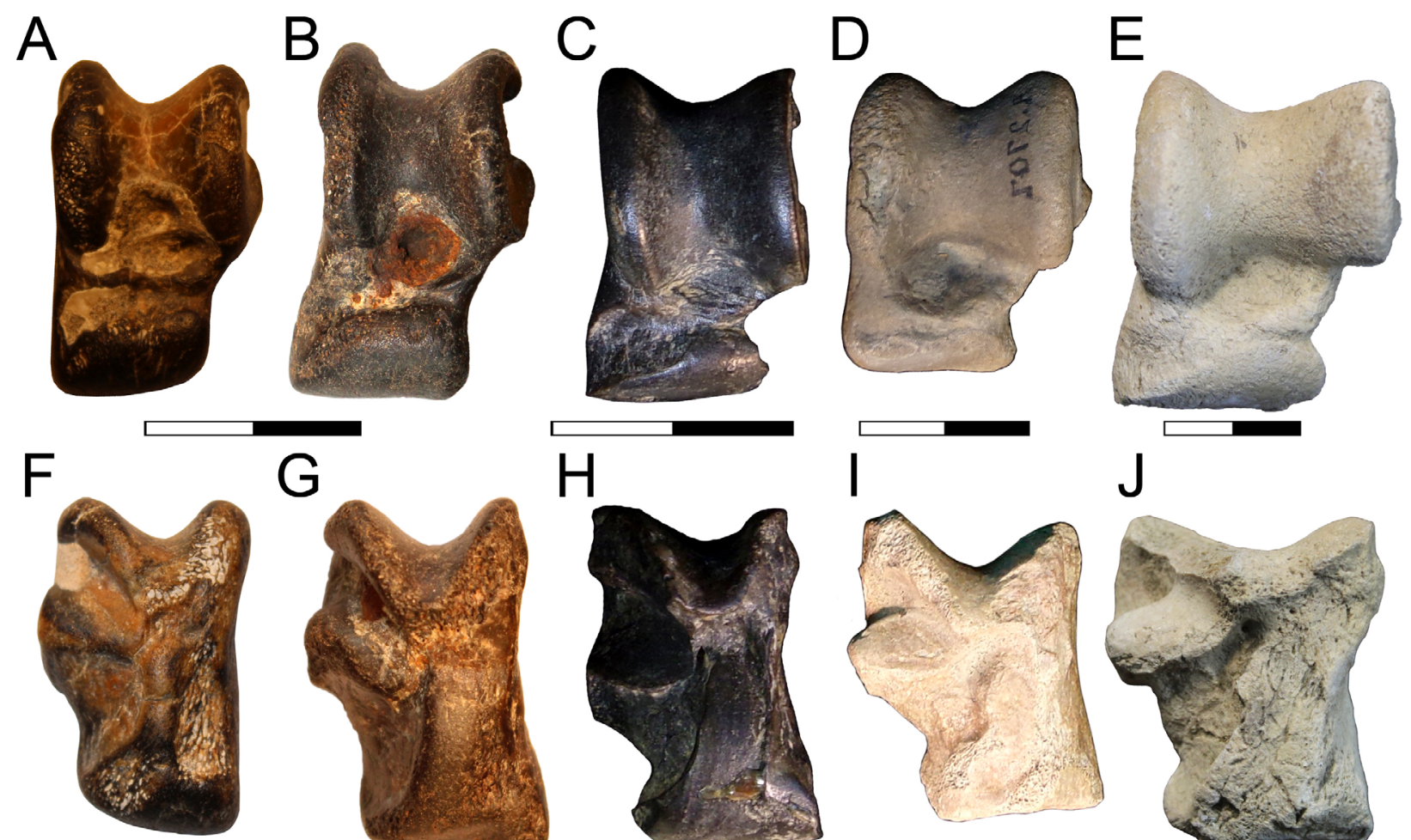

G
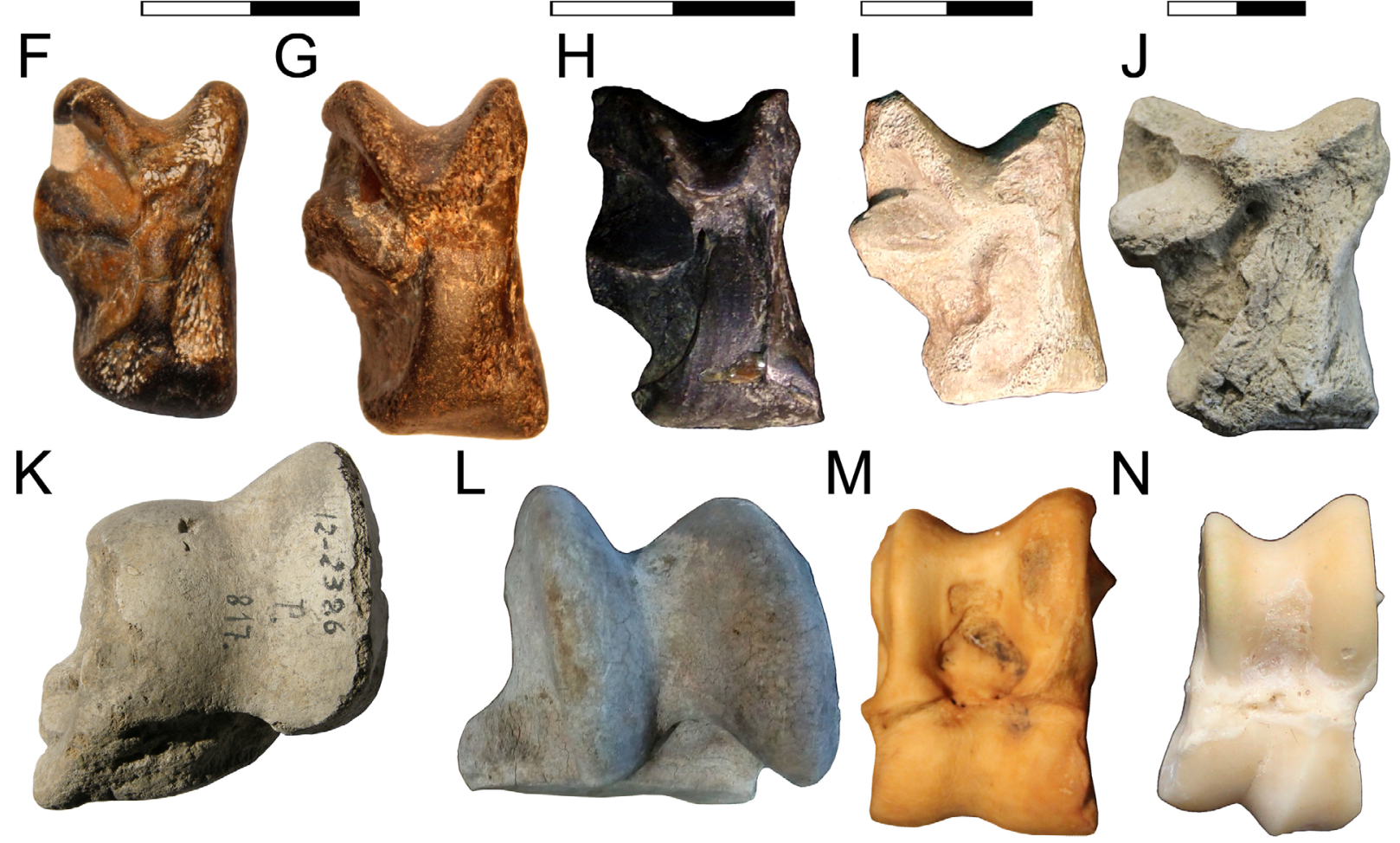

$\mathrm{N}$
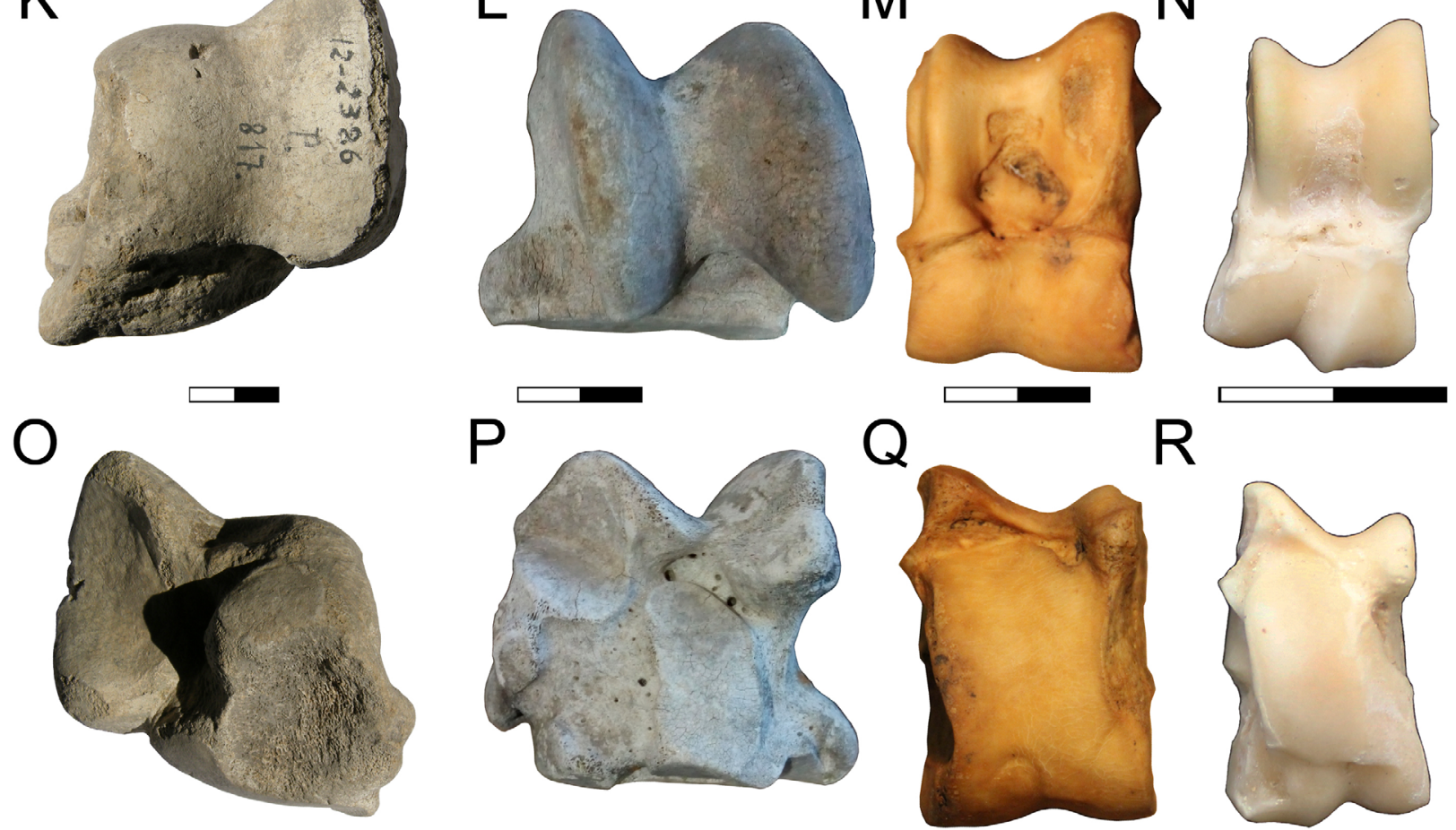

Figura 5. Figura comparativa de astrágalos del lado izquierdo de ungulados selectos en vista dorsal (A-E, K-N) y plantar (F-J, O-R). A, F, MMA 0050; B, G, MLP 17-II-18-25; C, H, Tetramerorhinus lucarius (MACN A-3009-15) (Proterotheriidae, Litopterna); D, I, Theosodon gracilis (MACN A-2707, revertido) (Macraucheniidae, Litopterna); E, J, Coniopternium andinum (MACN-Pv 11700, revertido) (Macraucheniidae, Litopterna); K, O, Toxodon sp. (MLP 12-2386) (Toxodontidae, Notoungulata); L, P, Equus caballus (colección personal NRC) (Equidae, Perissodactyla); M, Q, Blastocerus dichotomus (MACN-Ma 27.218, revertido) (Cervidae, Artiodactyla); N, R, Pecari tajacu (CFA-MA 14175, revertido) (Tayassuidae, Artiodactyla). Escalas $=20 \mathrm{~mm}$.

Figure 5. Comparative figure of left side astragali of selected ungulates in dorsal (A-E, K-N) and plantar (F-J, O-R) views. A, F, MMA 0050; B, G, MLP 17-II-18-25; C, H, Tetramerorhinus lucarius (MACN A-3009-15) (Proterotheriidae, Litopterna); D, I, Theosodon gracilis (MACN A-2707, reversed) (Macraucheniidae, Litopterna); E, J, Coniopternium andinum (MACN-Pv 11700, reversed) (Macraucheniidae, Litopterna); K, O, Toxodon sp. (MLP 12-2386) (Toxodontidae, Notoungulata); L, P, Equus caballus (personal colection NRC) (Equidae, Perissodactyla); M, Q, Blastocerus dichotomus (MACN-Ma 27.218, reversed) (Cervidae, Artiodactyla); N, R, Pecari tajacu (CFA-MA 14175, reversed) (Tayassuidae, Artiodactyla). Scale bars $=20 \mathrm{~mm}$. 
Corona et al., 2019a, b). En este sentido, los hallazgos aquí registrados concuerdan con la propuesta reciente de Ghizzoni et al. (2020) quienes sostienen hábitos pastadores para los Proterotheriidae del Pleistoceno.

\section{AGRADECIMIENTOS}

Agradecemos a D.H. Gambetta el acceso a las colecciones y el apoyo brindado durante la confección del presente manuscrito. A S. Bogan y E. Guerrero por sus valiosos comentarios y discusiones con respecto a la posición estratigráfica de los fósiles aquí reportados. Asimismo, agradecemos a M.D. Ezcurra, L. Chornogubsky, P. Teta, G. Cassini, S. Lucero y S. Bogan por permitirnos el acceso a las colecciones bajo su cargo.

\section{REFERENCIAS}

Álvarez, B.B. 1974. Los mamíferos fósiles del Cuaternario de Arroyo Toropí, Corrientes (Argentina). Ameghiniana, 11:295-311.

Ameghino, F. 1887. Enumeración sistemática de las especies de mamíferos fósiles coleccionados por Carlos Ameghino en los terrenos eocenos de Patagonia austral y depositados en el Museo de La Plata. Boletín del Museo de La Plata, 1:1-26.

Bond, M.; Cerdeño, E. \& López, G. 1995. Los ungulados nativos de América del Sur. In: M.T. Alberdi; G. Leone \& E.P. Tonni (eds.) Evolución biológica y climática de la Región Pampeana durante los últimos cinco millones de años, Consejo Superior de Investigaciones Científicas, p. 259-275.

Bond, M.; Perea, D.; Ubilla, M. \& Tauber, A. 2001. Neolicaphrium recens Frenguelli, 1921, the only surviving Proterotheriidae (Litopterna, Mammalia) into the South American Pleistocene. Paleovertebrata, 30:37-50.

Carrillo, J.D.; Amson, E.; Jaramillo, C.; Sánchez, R.; Quiroz, L.; Cuartas, C.; Rincón, A.F. \& Sanchez-Villagra, M.R. 2018.The Neogene Record of Northern South American Native Ungulates. Smithsonian Contributions to Paleobiology, 101:1-67. doi:10.5479/si.1943-6688.101

Cifelli, R.L. 1983. The origin and affinities of the South American Condylarthra and early Tertiary Litopterna (Mammalia). American Museum Novitates, 2772:1-49.

Cione, A.L. \& Tonni, E.P. 2005. Bioestratigrafía basada en mamíferos del Cenozoico superior de la provincia de Buenos Aires, Argentina. Geología y Recursos Minerales de la provincia de Buenos Aires, 11:183-200.

Cione, A.L.; Tonni, E.P., \& Dondas, A. 2005. A mastodont (Mammalia, Gomphotheriidae) from the Argentinian continental shelf. Neues Jahrbuch für Geologie und Paläontologie, 10:614630. doi:10.1127/njgpm/2005/2005/614

Corona, A.; Perea, D. \& Ubilla, M. 2019a. A new genus of Proterotheriinae (Mammalia, Litopterna) from the Pleistocene of Uruguay. Journal of Vertebrate Paleontology, 39: e1567523. doi:10.1080/02724634.2019.1567523

Corona, A.; Ubilla, M. \& Perea, D. 2019b. New records and diet reconstruction using dental microwear analysis for Neolicaphrium recens Frenguelli, 1921 (Litopterna, Proterotheriidae). Andean Geology, 46:153-157. doi:10.5027/andgeoV46n1-3136

Croft, D.; Gelfo, J. \& López, G. 2020. Splendid Innovation: the Extinct South American Native Ungulates. Annual Review of
Earth and Planetary Sciences, 48:1-32. doi: 10.1146/annurevearth-072619-060126

Frenguelli, J. 1921. Sobre un proterotérido del Pampeano superior de Córdoba, Neolicaphrium recens, nov. Actas de la Academia Nacional de Ciencias en Córdoba, 7:7-23.

Gaudioso, P.J.; Gasparini, G.M.; Herbst, R. \& Bárquez, R.M. 2017. First record of the Neolicaphrium recens Frenguelli, 1921 (Mammalia, Litopterna) in the Pleistocene of Santiago del Estero Province, Argentina. Papéis Avulsos de Zoologia, 57:23-29. doi:10.11606/0031-1049.2017.57.03

Ghizzoni, M.; Corona, A.; Ubilla, M. \& Perea, D. 2020. Estimación de masa corporal de proterotéridos del Cuaternario (Mammalia, Litopterna) y descripción de un nuevo ejemplar de Neolicaphrium recens Frenguelli, 1921. Revista Brasileira de Paleontologia, 23:153-162. doi:10.4072/rbp.2020.2.06

Kraglievich, L. 1934. La antigüedad pliocena de las faunas de Monte Hermoso y Chapadmalal, deducidas de su comparación con las que le precedieron y sucedieron. Montevideo, El Siglo Ilustrado, $136 \mathrm{p}$.

Luna, C.A.; Schmidt, G.I. \& Ochoa, J.G. 2015. Nuevos registros de Neolicaphrium recens Frenguelli (Litopterna, Proterotheriidae) procedentes de Corralito (Pleistoceno tardio-Holoceno temprano), Cordoba. Argentina. Ameghiniana, 53:20.

Perea, D.; Ubilla, M. \& Bond, M. 1995. The Proterotheriidae (Litopterna), surviving ungulates in the Pleistocene. In: JORNADAS ARGENTINAS DE PALEONTOLOGÍA DE VERTEBRADOS, 11, 1995. Libro de Resúmenes, Tucumán, p. 25.

Pomi, L.H. 2008. Una nueva asociación de vertebrados fósiles de Edad Ensenadense (Plioceno tardío-Pleistoceno medio) de la provincia de Buenos Aires, Argentina. Ameghiniana, 45:503-510.

Scherer, C.S.; Gregis Pitana, V. \& Ribeiro, A.M. 2009. Proterotheriidae and Macraucheniidae (Litopterna, Mammalia) from the Pleistocene of Rio Grande do Sul State, Brazil. Revista Brasileira de Paleontologia, 12:231-246. doi:10.4072/rbp.2009.3.06

Schmidt, G.I. 2015. Actualización sistemática y filogenia de los Proterotheriidae (Mammalia, Litopterna) del "Mesopotamiense" (Mioceno Tardío) de Entre Ríos, Argentina. Revista Brasileira de Paleontologia, 18:521-546. doi:10.4072/rbp.2015.3.14

Schmidt, G.I.; Luna, C.A. \& Vezzosi, R.I. 2016. Avances en el conocimiento de los elementos postcraneales de Neolicaphrium Frenguelli, 1921 (Litopterna, Proterotheriidae). In: CONGRESO LATINOAMERICANO DE PALEONTOLOGÍA, 9, 2016. Libro de Resúmenes, Lima, p. 149.

Scott, W.B. 1910. Part I: Litopterna of the Santa Cruz beds. In: W.B. Scott (ed.) Reports of the Princeton University Expedition to Patagonia, 1896-1899, Volume III Paleontology 7, J. Pierpont Morgan Publication Fund, 20 pls. +156 p.

Soibelzon, E.; Tonni, E.P. \& Bidegain, J.C. 2008. Cronología, magnetoestratigrafía y caracterización bioestratigráfica del Ensenadense (Pleistoceno inferior-medio) en la ciudad de Buenos Aires. Revista de la Asociación Geológica Argentina, 63:421-429.

Soria, M. 2001. Los Proterotheriidae (Litopterna, Mammalia), sistemática, origen y filogenia. Monografías del Museo Argentino de Ciencias Naturales, 1:1-167.

Tauber, A. 2000. Hallazgos de Proterotheriidae (Mammalia, Litopterna) en el Pleistoceno de Córdoba, Argentina. Ameghiniana, 37:157-162.

Tonni, E.P. \& Cione, A.L. 1999. Pleistocene continental vertebrates from the present marine shelf of Argentina. Current Research in the Pleistocene, 16:134-136. 
Ubilla, M.; Perea, D.; Bond, M. \& Rinderknecht, A. 2011. The first cranial remains of the Pleistocene proterotheriid Neolicaphrium Frenguelli, 1921 (Mammalia, Litopterna): a comparative approach. Journal of Vertebrate Paleontology, 31:193-201. doi:10.1080/02724634.2011.539647

Vezzosi, R.I. 2016. Diversidad de los mamíferos del Pleistoceno de la Provincia de Santa Fe, Argentina: aspectos taxonómicos, bioestratigráficos y paleobiogeográficos relacionados. Mastozoología Neotropical, 23:215-217.

Vezzosi, R.; Schmidt, G. \& Brunetto, E. 2009. Un Proterotheriinae (Proterotheriidae: Litopterna) en el Pleistoceno tardíoHoloceno temprano (Lujanense) de Santa Fé. In: JORNADAS ARGENTINAS DE PALEONTOLOGÍA DE VERTEBRADOS, 24, 2009. Libro de Resúmenes, San Rafael, p. 62.
Villafañe, A.L.; Ortiz-Jaureguizar, E. \& Bond, M. 2006. Cambios en la riqueza taxonómica y en las tasas de primera y última aparición de los Proterotheriidae (Mammalia, Litopterna) durante el Cenozoico. Estudios Geológicos, 62:155-166.

Violante, R.A. \& Parker, G. 2004. The post-Last Glacial Maximum transgression in the de la Plata river and adjacent Inner Continental Shelf, Argentina. Quaternary International, 114:167-181. doi:10.1016/S1040-6182(03)00036-3

Received in 30 October, 2019; accepted in 25 September, 2020. 